\title{
A INFLUÊNCIA DA PRÁTICA DO PILATES NA QUALIDADE DE VIDA DOS IDOSOS: ESTUDO CLINICO E RANDOMIZADO
}

Fabiana Roveda Nery

Kátia Cristina Ugolini Mugnol²

Vivian Bertoni Xavier ${ }^{3}$

Vera Lúcia dos Santos Alves ${ }^{4}$

resumo

O treinamento físico vem se mostrando efetivo para minimizar as consequências deletérias do processo de envelhecimento. O Pilates, porém, ainda não foi foco de estudos controlados em idosos e, assim, objetivamos avaliar o impacto do método Pilates na qualidade de vida de indivíduos na terceira idade. Participaram deste estudo prospectivo e randomizado 44 voluntários acima de 60 anos, de

1 Graduada em Fisioterapia. Mestre em Ciência e Tecnologia em Saúde. Fisioterapeuta. E-mail: fabirnery@yahoo.com.br

2 Graduada em Biomedicina. Doutora em Biotecnologia. Coordenadora e Professora do curso de Mestrado Profissional em Ciência e Tecnologia em Saúde na Universidade de Mogi das Cruzes. E-mail: katiac@umc.br

3 Graduada em Fisioterapia. Doutora em Ciências da Saúde. Professora Convidada da Faculdade de Ciências Médicas da Santa Casa de São Paulo. E-mail: vivianbxavier@hotmail.com

4 Graduada em Fisioterapia. PhD em Ciências da Saúde. Professora Adjunta da Faculdade de Ciências Médicas da Santa Casa de São Paulo e da Universidade de Mogi das Cruzes. E-mail: fisioterapiasc@uol.com.br 
ambos os sexos, divididos em dois grupos: controle (22 idosos que não realizaram exercícios) e intervenção (22 idosos que realizaram um protocolo constituído por exercícios com o método Pilates). 0 protocolo no grupo intervenção foi aplicado por um fisioterapeuta, duas vezes por semana, com sessões de 60 minutos mantidas por dois meses. Para análise da qualidade de vida, foram aplicados, antes e após o protocolo, os questionários WHOQOL-bref e WHOQOL- old para ambos os grupos. Após a realização dos dois meses de protocolo, pudemos observar o aumento da pontuação de todos os domínios dos questionários de qualidade de vida no grupo de intervenção, concluindo que o protocolo pode ser um produto importante influenciando positivamente a qualidade de vida da população idosa submetida ao método Pilates.

palavras-chave

Idoso. Qualidade de Vida. Exercício. Técnicas de Exercício e de Movimento.

\section{Introdução}

A maior concentração de idosos na população comumente vem associada ao aumento na incidência de doenças crônico-degenerativas, que podem ser acompanhadas por sequelas, limitando o desempenho funcional em relação à incapacidade precoce (TAVARES; DIAS, 2012). O envelhecimento por si só vem acompanhado de alterações fisiológicas nos sistemas ósseo, muscular e articular, diminuindo funções locomotoras (HERNANDES; BARROS, 2004). Hoje, porém, é comprovado que quanto mais ativa é a pessoa, menos limitações físicas ela tem. A prática de exercícios regular e sistematizada tem papel na proteção da capacidade funcional em todas as idades, com o foco na atualidade, sendo maior aos idosos (FRANCHI; MONTENEGRO JUNIOR, 2005).

O tipo de exercício físico recomendado para a terceira idade no passado era o aeróbio pelos seus efeitos no sistema cardiovascular e benefícios psicológicos (FRANCHI; MONTENEGRO JUNIOR, 2005). Entretanto, nos últimos anos tem sido demonstrada também a necessidade do treinamento com exercício resistido para incremento de força e resistência (POLLOCK et al., 2000). Dentre as formas de treinamento contra a resistência, o método Pilates surge como forma de condicionamento físico particularmente interessado em proporcionar bem-estar geral ao indivíduo, melhorando força, flexibilidade, postura, controle, consciência corporal e percepção do movimento (BLUM, 2002). 
Além dos benefícios, os protocolos de exercício em idosos também têm influenciado na qualidade de vida e na manutenção da capacidade funcional, gera impacto positivo na autonomia dos idosos, que pode ser percebida no desempenho das atividades de vida diária (GALISTEU et al., 2006). Essas atividades possibilitam que a pesquisa clínica possa investigar o papel de uma modalidade especifica de treinamento nos idosos e a sua qualidade de vida correlacionada à atividade física. Sendo assim, objetivamos verificar o impacto de um protocolo de exercício físico focado no método Pilates na qualidade de vida de idosos.

\section{Casuística e método}

Este estudo prospectivo foi randomizado por meio de sorteio por envelopes selados que dividiram os 48 voluntários, acima de 60 anos, de ambos os gêneros, em dois grupos, controle e intervenção, após a aprovação do Comitê de Ética em Pesquisa sob CAEE: 09163213.3.0000.5497, conforme Figura 1.

Figura 1 - Fluxograma apresentando o recrutamento e alocação dos 48 idosos abordados para participação no estudo.

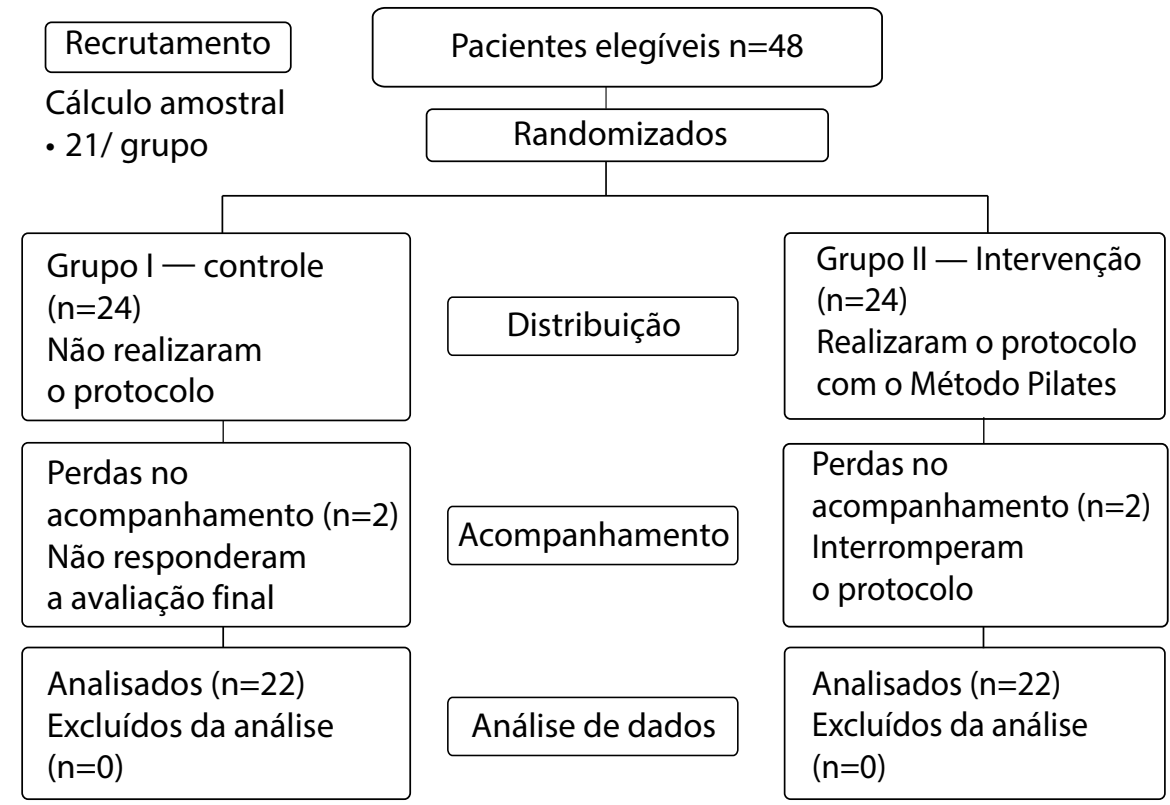

Fonte: SCHULZ; ALTMAN; MOHER (2010). 
Foram incluídos idosos com idade acima de 60 anos que concordaram em participar da pesquisa assinando o termo de consentimento livre e esclarecido, todos sem alterações cognitivas, que não praticavam outra modalidade de atividade física orientada e não possuíam limitações físicas para a realização dos exercícios propostos no estudo. Foram excluídos os indivíduos com doenças prévias, agudas ou crônicas, ortopédicas e/ou neurológicas, hipertensos, diabéticos e cardiopatas não controlados ou, ainda, aqueles que foram submetidos a qualquer procedimento cirúrgico a menos de seis meses antes do protocolo.

Todos os voluntários foram avaliados com o apoio de uma ficha de avaliação contendo dados pessoais e antropométricos. Os idosos ainda responderam aos questionários WHOQOL-bref e WHOQOL-old de forma individual, na avaliação e também no momento da reavaliação. O WHOQOL-bref avalia a qualidade de vida em adultos por meio de 26 questões. Cada questão é respondida com uma pontuação que varia de 1 a 5, sendo que o somatório das respostas pode ser avaliado em conjunto ou em quatro domínios: físico, psicológico, relações sociais e meio ambiente (THE WHOQOL GROUP, 1998).

O WHOQOL-old é um questionário complementar que deve ser aplicado em conjunto com o WHOQOL-bref quando o objetivo é a avaliação da qualidade de vida em idosos. O WHOQOL-old é composto por 24 questões secionadas em seis facetas, sendo elas: funcionamento dos sentidos, autonomia, atividades passadas, presentes e futuras, participação social, morte/morrer, e intimidade. Cada questão permite ao paciente classificar a intensidade de sua situação, e para cada resposta há uma pontuação de 1 a 5 (THE WHOQOL GROUP, 1998).

O grupo I, que não realizou o protocolo, foi avaliado e orientado sobre a importância da realização de atividades físicas para o bem-estar e a saúde física, em uma palestra educativa, sendo reavaliado após passados dois meses. Os 22 pacientes do grupo II realizaram o protocolo de dois meses de exercícios com toda a sequência das atividades propostas, sendo executadas em todas as sessões para a padronização de todos os atendimentos já que o conjunto dos pacientes era dividido em grupos de até cinco idosos, que realizaram as sessões, duas vezes por semana, com cada sessão durando 60 minutos.

Os exercícios foram realizados sempre duas horas após refeições, sendo respeitado o período de 48 horas de repouso entre uma sessão e outra. Todos os exercícios foram realizados em solo utilizando acessórios como colchonetes, bola e faixas elásticas, sempre orientados por um mesmo fisioterapeuta demonstrando e monitorando a execução. A primeira sessão de exercício foi realizada para familiarização dos idosos quanto à técnica, orientações gerais e aprendizado da monitoração da pressão arterial e frequência cardíaca, que era aferida antes e após todas as sessões. 
O protocolo foi baseado no método Pilates e incluiu exercícios em uma sequência que era realizada sempre na mesma ordem, sendo descrita a seguir: com os pacientes sedestados foi feito o alongamento de tronco com torção da coluna e serrote. Em decúbito dorsal, foram executados os exercícios de fortalecimento de cintura pélvica e ponte. Para o fortalecimento das musculaturas abdominal e paravertebral, foram executados movimentos com a posição $\mathrm{V}$, elevação e abdução de perna, mergulho de cisne, nadando, e o aperto de calcanhar em decúbito ventral. Para membros superiores, com os pacientes em ortostatismo, foram executados o fortalecimento de bíceps, anjo em pé e fortalecimento de costas. Todos os exercícios foram repetidos por dez vezes cada um.

Para análise dos dados, utilizamos o programa SPSS 22.0, com aplicação do teste U Mann-Whitney para variáveis não paramétricas, pré e pós-intervenção, dentro dos domínios dos questionários comparando os dois grupos e Wilcoxon para análise dentro de cada grupo nos dois momentos. O nível de significância foi de 0,05 .

\section{Resultados}

Foram avaliados os 44 idosos, 22 constituíram o grupo I (controle), com média de idade de 64,22 anos $( \pm 3,13)$, sendo 18 do gênero feminino e 4 do masculino. No grupo II (intervenção), a média de idade foi de 65,57 anos $( \pm 4,39)$, com 17 pacientes sendo do gênero feminino e 5 do masculino.

Na comparação inicial entre os grupos, foi observada heterogeneidade somente para o domínio meio ambiente do questionário WHOQOL-bref, com $p$ 0,008. Para esse domínio, o grupo intervenção apresentou pontuação média de $2,85( \pm 0,15)$ e o controle, $3,02( \pm 0,23)$, demonstrando a pior condição nesse domínio para o grupo intervenção antes do protocolo.

$\mathrm{Na}$ análise isolada da evolução do grupo I (controle), comparando-se as avaliações inicial e final, observa-se que somente o domínio físico do questionário WHOQOL-bref mostrou alteração, $\operatorname{com} p<0,010$ demonstrando aumento da pontuação média na reavaliação, que foi de 2,66 $( \pm 0,14)$ na avaliação inicial para 2,78 $( \pm 0,10)$ na reavaliação. Para o WHOQOL-old no grupo controle, observamos a diminuição na pontuação de quase todos domínios no período analisado, com menores valores médios evidenciados na reavaliação conforme demonstrado na Tabela 1, que aponta também o $p$ valor da comparação entre o período de avaliações inicial e final do WHOQOL-old no grupo I, demonstrando a menor pontuação após o período de estudo. 
Tabela 1 - Apresentação de todos os domínios do questionário WHOQOL-old na comparação entre as avaliações inicial e final no grupo I (controle) dos 22 pacientes incluídos na amostra.

\begin{tabular}{|c|c|c|c|c|c|c|}
\hline Domínios - WHOQOL-old - grupo I & $\mathrm{N}$ & média & DP & mínimo & máximo & $p$ \\
\hline Habilidade sensorial - inicial & 22 & 3,49 & 0,45 & 2,50 & 4,25 & \multirow{2}{*}{0,012} \\
\hline Habilidade sensorial - final & 22 & 3,33 & 0,42 & 2,50 & 4,50 & \\
\hline Autonomia - inicial & 22 & 3,23 & 0,77 & 1,75 & 4,50 & \multirow{2}{*}{0,016} \\
\hline Autonomia - final & 22 & 3,09 & 0,68 & 1,75 & 4,00 & \\
\hline Atividade pas/pre/fut - inicial & 22 & 3,34 & 0,98 & 1,25 & 4,75 & \multirow{2}{*}{0,016} \\
\hline Atividade pas/pre/fut - final & 22 & 3,22 & 0,90 & 1,25 & 4,25 & \\
\hline Participação social - inicial & 22 & 3,68 & 0,74 & 2,00 & 5,00 & \multirow{2}{*}{0,102} \\
\hline Participação social - final & 22 & 3,60 & 0,68 & 2,00 & 4,50 & \\
\hline Morte/morrer - inicial & 22 & 3,14 & 0,94 & 1,00 & 4,75 & \multirow{2}{*}{0,317} \\
\hline Morte/morrer - final & 22 & 3,16 & 0,89 & 1,50 & 4,75 & \\
\hline Intimidade - inicial & 22 & 3,07 & 0,63 & 1,75 & 4,00 & \multirow{2}{*}{0,157} \\
\hline Intimidade - final & 22 & 3,05 & 0,60 & 1,75 & 3,75 & \\
\hline Total - inicial & 22 & 3,32 & 0,40 & 2,46 & 3,79 & \multirow{2}{*}{$\begin{array}{c}< \\
0,001\end{array}$} \\
\hline Total - final & 22 & 3,24 & 0,36 & 2,38 & 3,75 & \\
\hline
\end{tabular}

Legenda - n: número de pacientes; DP: desvio-padrão; p: nível de significância; Atividade pas/pre/ fut: atividade passado, presente e futuro.

Quando analisamos isoladamente o grupo II (intervenção), todos os domínios do questionário WHOQOL-bref aumentaram significativamente, tendo $p<0,001$ em todos os domínios avaliados, conforme demonstra a Tabela 2. 
Tabela 2 - Pontuação do grupo II (intervenção) submetido ao tratamento com o método Pilates para os domínios de qualidade de vida, saúde, físico, psicológico, relações sociais e meio ambiente do questionário WHOQOL-bref entre os momentos inicial e final.

\begin{tabular}{|c|c|c|c|c|c|c|}
\hline Domínios - WHOQOL-bref - grupo II & $\mathrm{N}$ & média & DP & mínimo & máximo & $p$ \\
\hline Qualidade de vida - inicial & 22 & 2,36 & 0,90 & 1,00 & 4,00 & \multirow{2}{*}{$<0,001$} \\
\hline Qualidade de vida - final & 22 & 3,95 & 0,49 & 3,00 & 5,00 & \\
\hline Saúde - inicial & 22 & 2,14 & 0,47 & 2,00 & 4,00 & \multirow{2}{*}{$<0,001$} \\
\hline Saúde - final & 22 & 4,00 & 0,44 & 3,00 & 5,00 & \\
\hline Físico - inicial & 22 & 2,70 & 0,16 & 2,43 & 3,00 & \multirow{2}{*}{$<0,001$} \\
\hline Físico - final & 22 & 4,05 & 0,11 & 3,86 & 4,29 & \\
\hline Psicológico - inicial & 22 & 2,59 & 0,20 & 2,17 & 3,00 & \multirow{2}{*}{$<0,001$} \\
\hline Psicológico - final & 22 & 4,06 & 0,22 & 3,50 & 4,33 & \\
\hline Relações sociais - inicial & 22 & 2,88 & 0,22 & 2,67 & 3,33 & \multirow{2}{*}{$<0,001$} \\
\hline Relações sociais - final & 22 & 4,65 & 0,22 & 4,33 & 5,00 & \\
\hline Meio ambiente - inicial & 22 & 2,85 & 0,15 & 2,63 & 3,25 & \multirow{2}{*}{$<0,001$} \\
\hline Meio ambiente - final & 22 & 3,48 & 0,14 & 3,25 & 3,75 & \\
\hline
\end{tabular}

Para o questionário WHOQOL-old, os resultados do grupo intervenção nos momentos pré e pós-tratamento mostraram significância nos domínios habilidade sensorial, autonomia, morte/morrer e intimidade, com aumento significativo na qualidade de vida com $p$ valor apresentado na Tabela 3. 
Tabela 3 - Valores dos domínios do questionário WHOQOL-old na comparação entre os momentos inicial e final no grupo II (intervenção) após o tratamento dos 22 pacientes incluídos.

\begin{tabular}{|c|c|c|c|c|c|c|}
\hline Domínios & $\mathbf{N}$ & média & DP & mínimo & máximo & $p$ \\
\hline Habilidade sensorial - inicial & 22 & 3,72 & 0,59 & 2,50 & 4,50 & \multirow{2}{*}{0,018} \\
\hline Habilidade sensorial - final & 22 & 4,11 & 0,82 & 2,00 & 5,00 & \\
\hline Autonomia - inicial & 22 & 3,44 & 0,66 & 1,75 & 4,50 & \multirow{2}{*}{0,009} \\
\hline Autonomia - final & 22 & 3,88 & 0,38 & 3,25 & 4,75 & \\
\hline Atividade pas/pre/fut - inicial & 22 & 3,75 & 0,67 & 2,00 & 5,00 & \multirow{2}{*}{0,874} \\
\hline Atividade pas/pre/fut - final & 22 & 3,80 & 0,57 & 2,75 & 5,00 & \\
\hline Participação social - inicial & 22 & 3,56 & 0,44 & 2,50 & 4,25 & \multirow{2}{*}{0,256} \\
\hline Participação social - final & 22 & 3,74 & 0,64 & 2,50 & 5,00 & \\
\hline Morte/morrer - inicial & 22 & 3,26 & 0,98 & 1,00 & 4,75 & \multirow{2}{*}{0,001} \\
\hline Morte/morrer - final & 22 & 3,92 & 0,89 & 2,00 & 5,00 & \\
\hline Intimidade - inicial & 22 & 3,25 & 0,61 & 1,75 & 4,25 & \multirow{2}{*}{0,001} \\
\hline Intimidade - final & 22 & 4,02 & 0,45 & 3,25 & 5,00 & \\
\hline Total - inicial & 22 & 3,50 & 0,37 & 2,63 & 3,92 & \multirow{2}{*}{$<0,001$} \\
\hline Total - final & 22 & 3,91 & 0,38 & 3,21 & 4,79 & \\
\hline
\end{tabular}

Legenda - n: número de pacientes; DP: desvio-padrão; p: nível de significância; Atividade pas/pre/ fut: atividade passado, presente e futuro.

Na comparação da avaliação final entre os grupos intervenção e controle, observamos significância estatística apenas para os domínios do questionário WHOQOL-bref, com $p<0,001$ após o período analisado, ratificando o aumento da qualidade de vida do grupo intervenção, conforme Tabela 4. 
Tabela 4 - Comparação entre o grupo intervenção e controle para a pontuação final para todos os domínios do questionário WHOQOL-bref ao final do estudo.

\begin{tabular}{|c|c|c|c|c|c|}
\hline Domínios & Grupos & $\mathrm{N}$ & média & DP & $p$ \\
\hline \multirow{3}{*}{ Qualidade de vida } & intervenção & 22 & 3,95 & 0,49 & \multirow{2}{*}{$<0,001$} \\
\hline & controle & 22 & 2,91 & 0,75 & \\
\hline & Total & 44 & 3,43 & 0,82 & \\
\hline \multirow{3}{*}{ Saúde } & intervenção & 22 & 4,00 & 0,44 & \multirow{2}{*}{$<0,001$} \\
\hline & controle & 22 & 2,05 & 0,21 & \\
\hline & Total & 44 & 3,02 & 1,05 & \\
\hline \multirow{3}{*}{ Físico } & intervenção & 22 & 4,05 & 0,11 & \multirow{2}{*}{$<0,001$} \\
\hline & controle & 22 & 2,78 & 0,18 & \\
\hline & Total & 44 & 3,41 & 0,66 & \\
\hline \multirow{3}{*}{ Psicológico } & intervenção & 22 & 4,06 & 0,22 & \multirow{2}{*}{$<0,001$} \\
\hline & controle & 22 & 2,64 & 0,17 & \\
\hline & Total & 44 & 3,35 & 0,74 & \\
\hline \multirow{3}{*}{ Relações sociais } & intervenção & 22 & 4,65 & 0,22 & \multirow{2}{*}{$<0,001$} \\
\hline & controle & 22 & 2,94 & 0,37 & \\
\hline & Total & 44 & 3,80 & 0,92 & \\
\hline \multirow{3}{*}{ Meio ambiente } & intervenção & 22 & 3,48 & 0,14 & \multirow{2}{*}{$<0,001$} \\
\hline & controle & 22 & 3,05 & 0,23 & \\
\hline & Total & 44 & 3,26 & 0,29 & \\
\hline
\end{tabular}


O estudo evidenciou resultado positivo na qualidade de vida dos indivíduos do grupo de intervenção após a prática de dois meses de um protocolo de exercícios de Pilates. Esse resultado se assemelha ao encontrado por Gladwell et al. (2006), que atribui ao Pilates o benefício de potencializar a saúde geral, a flexibilidade e a propriocepção em indivíduos com dor lombar crônica. Apesar de nosso estudo ter focado na qualidade de vida ao invés da dor, acreditamos que a melhora da primeira, demonstrada na pontuação dos questionários utilizados, seja a tradução de um quadro clínico geral mais estável e favorável na população idosa que participou de nossa pesquisa.

Em estudo idealizado por Siqueira Rodrigues et al. (2010), houve a melhora significativa na autonomia pessoal, no equilíbrio estático e na qualidade de vida de mulheres idosas com a prática do método Pilates. Esse achado foi reproduzido em nosso estudo, porém envolvemos pacientes de ambos os gêneros nos grupos tanto de intervenção como no de controle, o que permitiu que generalizássemos o resultado para homens e mulheres, obtendo melhora dos domínios relativos à qualidade de vida em todos os pacientes do grupo de intervenção independentemente de gênero. Essa melhora avaliada pelo WHOQOL-old e pelo WHOQOL-bref é coligada ao conjunto de resultados de estudo realizado por Matsudo, Matsudo e Barros Neto (2001), que mostraram evolução da aptidão física e da capacidade funcional após programa de exercício, fortalecendo a hipótese de que a prática regular de atividade física é instrumento para a prevenção e promoção da saúde em idosos, grupo contemplado em nossa amostra.

A associação entre níveis de atividade física e depressão pode fundamentar uma explicação para o comportamento do grupo de controle em nossa pesquisa. Observamos, no período estudado, que os idosos que somente foram orientados sobre a prática de atividade física, sem a inclusão em um programa específico de condicionamento, mantiveram ou diminuíram seu escore nos questionários analisados, sugerindo uma piora na qualidade de vida em comparação aos idosos que praticaram o Pilates. Essa observação encontra paralelo na literatura com Minghelli et al. (2013) com os pesquisadores já verificando relação significante entre os níveis de atividade física e o estado de saúde mental, com menor prevalência de indicadores de depressão para idosos não sedentários, igualmente aos achados em nosso estudo.

Quando isolamos a discussão nos domínios dos questionários apresentados pelos dois grupos estudados, nota-se a melhora da mobilidade, energia e capacidade para realizar atividades de vida diária no grupo de intervenção, 
com essa mesma afirmação sendo encontrada no estudo de Irez et al. (2011), que demonstram que exercícios de Pilates são eficazes para o equilíbrio dinâmico, a flexibilidade, o tempo de reação e a força muscular, bem como diminuem a propensão a quedas em mulheres idosas. Nossa pesquisa não avaliou o risco de queda, porém o aumento do domínio de habilidade sensorial e da autonomia denota incremento da mobilidade e provável impacto positivo na diminuição das quedas.

Nossos resultados confirmam ainda os achados do estudo realizado por Mota et al. (2006), que avaliaram a qualidade de vida relacionada à saúde com o SF36 comparando dois grupos com mais de 65 anos. O grupo experimental foi composto por 46 pessoas que estavam envolvidos em programa de atividade física regular e demonstrou que a percepção de qualidade de vida associada à saúde se encontra intimamente ligada à prática formal da atividade. Observamos, então, em nossa pesquisa, a importância do protocolo de exercício ser praticado duas vezes por semana determinando o desfecho favorável da atividade sistematizada.

Diversos grupos de estudo buscam o direcionamento dos programas de treinamento físico e sua adequação a diversas faixas etárias, minimizando nos idosos o curso do envelhecimento sobre a função muscular e seu papel nas limitações funcionais com melhora na qualidade de vida. A grande questão é se todas as modalidades de exercício podem trazer benefícios e segurança aos praticantes, já que cada uma apresenta particularidades quanto a contração muscular e energia dispensada para a atividade (LACOURT; MARINI, 2006).

Rogers e Gibson (2009) investigaram as respostas de adultos submetidos ao Pilates idealizado no solo e outro grupo que realizava atividade aeróbia. Os dois protocolos foram mantidos por oito semanas com duração de 1 hora por sessão, repetida três vezes na semana. Nos pacientes que realizaram o treino aeróbio, houve a redução da gordura abdominal. Nos praticantes de Pilates, houve redução significativa da gordura corporal e da circunferência de tórax e braço com aumento do alongamento, efeitos creditados à junção de exercícios isométricos e isotônicos incluídos na rotina do Pilates.

Os exercícios da técnica do Pilates são, na sua maioria, executados na posição deitada, havendo diminuição do impacto nas articulações de sustentação do corpo e, principalmente, na coluna vertebral, permitindo recuperação rápida das estruturas musculares, articulares e ligamentares após a atividade (FERREIRA et al., 2007). Ao contrário dos exercícios de força tradicionais baseados no treino de músculos isolados, os exercícios de Pilates têm abordagem holística, o que requer ativação e coordenação de vários grupos musculares ao mesmo tempo (IREZ et al., 2011). A característica principal do método é o trabalho resistido 
e o alongamento dinâmico, realizados em conjunto com a respiração, respeitando a concentração, a centralização, a fluidez de movimento, a respiração, a precisão e o controle. O método visa o reforço dos músculos localizados no centro do corpo por permitir melhora globalizada (BROWN; FLOOD, 2013).

A melhora nesta pesquisa de vários domínios, incluindo o psicológico do grupo de intervenção, comparado ao controle, confirma o que dizem Brovold, Skelton e Bergland (2013), que o exercício físico provoca ativação cognitiva e atua no desempenho das habilidades sociais. A associação entre os maiores níveis de atividade física e menores de depressão nos idosos já está estabelecida na literatura (MINGHELLI et al., 2013). Os estudos correlacionam os níveis de atividade e o estado de saúde mental, com menor prevalência de indicadores de depressão para idosos não sedentários, igualmente ao achado em nosso estudo.

Podemos ainda comparar este trabalho ao de Penedo e Dahn (2005), que, a partir de uma revisão sistemática, objetivaram avaliar a relação entre o exercício, asaúde física e a mental. Segundo os autores, geralmente, os participantes envolvidos em atividade física regular exibem resultados de saúde mais desejáveis, incluindo a qualidade de vida, capacidade funcional e estado de humor, assim como em nossos resulados, em que os indivíduos submetidos ao exercício apresentaram maior qualidade de vida. Sendo assim e de acordo com estudos realizados por Phrompaet et al. (2011), um programa de exercícios promove mais mudanças qualitativas do que quantitativas, sendo sua adoção uma medida de segurança que deve ser incluída na rotina da população idosa, o que demonstra a importância de utilizarmos questionários de qualidade de vida em nosso estudo em detrimento de medidas quantitativas de atividade física.

\section{Conclusão}

Concluímos que houve melhora significativa na qualidade de vida dos idosos após a participação em um programa de exercícios com o método Pilates.

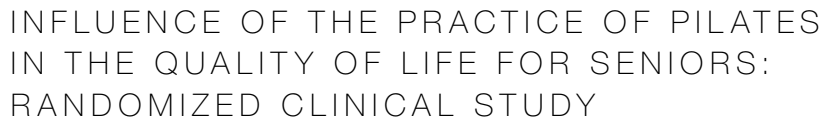

abstract

Physical training has proved effective to minimize the deleterious consequences of the aging process, but Pilates has not been the focus of controlled studies in the elderly so we aimed to evaluate the 
impact of the method in the life quality of this group. In this prospective randomized study, 44 volunteers over 60 years old, from both sexes, were divided into two groups: control (22 seniors who did not perform exercises) and intervention (22 elderly who underwent a protocol consisting of Pilates exercises). The protocol in the intervention group was deployed by the same physical therapist twice a week, 60-minute sessions during two months. To analyze the quality of life, it was administered to both groups, before and after the protocol, the WHOQOL-bref and old questionnaires. It could be observed increased scores for all domains of life quality for the intervention group and it was concluded that the protocol can be an important tool influencing positively the life quality of the elderly population undergoing Pilates.

keywords

Aged. Quality of Life. Exercise. Exercise Movement Techniques.

referências

BLUM, Charles L. Chiropractic and Pilates therapy for the treatment of adult scoliosis. Journal of Manipulative and Physiological Therapeutics, St. Louis, MO, v. 25, n. 4 p. E3, June 2002

BROVOLD, Therese; SKELTON, Dawn A.; BERGLAND, Astrid. Older Adults Recently Discharged from the Hospital: Effect of Aerobic Interval Exercise on Health-Related Quality of Life, Physical Fitness, and Physical Activity. Journal of the American Geriatrics Society, Malden, MA, v. 61, n. 9, p. 1580-1585, Sept. 2013.

BROWN, Cynthia J.; FLOOD, Kellie L. Mobility Limitation in the Older Patient: A Clinical Review. JAMA, Chicago, v. 310, n. 11, p. 1168-1177, Sept. 2013.

FERREIRA, Cristiane Bainchetti et al. O método Pilates $®$ sobre a resistência muscular localizada em mulheres adultas. Motricidade, Santa Maria da Feira, v. 3, n. 4, p. $76-81$, out. 2007.

FRANCHI, Kristiane Mesquita Barros; MONTENEGRO JUNIOR, Renan Magalhães. Atividade física: uma necessidade para a boa saúde na terceira idade. Revista Brasileira em Promoção da Saúde, Fortaleza, v. 18, n. 3, p. 152-156, 2005.

GALISTEU, Kátia J. et al. Qualidade de Vida de idosos de um grupo de convivência com a mensuração da escala de Flanagan. Arquivos de Ciências da Saúde, São José do Rio Preto, v. 13, n. 4, p. 209-214, out./dez. 2006.

GLADWELL, Valerie et al. Does a Program of Pilates Improve Chronic Non-Specific Low Back Pain? Journal of Sport Rehabilitation, Champaign, v. 15, n. 4, p. 338-350, Nov. 2006.

HERNANDES, Elizabeth Sousa Cagliari; BARROS, Jônatas de França. Efeitos de um programa de atividades físicas e educacionais para idosos sobre o desempenho em testes de atividades da vida diária. Revista Brasileira de Ciência e Movimento, Brasília, v. 12, n. 2, p. 43-50, jun. 2004. 
IREZ, Gonul Babayigit et al. Integrating Pilates Exercise into an Exercise Program for $65+$ Year-Old Women to Reduce Falls. Journal of Sports Science \& Medicine, Bursa, v. 10, n. 1, p. 105-11, Mar. 2011.

LACOURT, Marcelle Xavier; MARINI, Lucas Lima. Decréscimo da função muscular decorrente do envelhecimento e a influência na qualidade de vida do idoso: uma revisão de literatura. Revista Brasileira de Ciências do Envelhecimento Humano, Passo Fundo, v. 3, n. 1, p. 114-121, jan./jul. 2006.

MATSUDO, Sandra Mahecha; MATSUDO, Victor Keihan Rodrigues; BARROS NETO, Turíbio Leite. Atividade física e envelhecimento: aspectos epidemiológicos. Revista Brasileira de Medicina do Esporte, Niterói, v. 7, n. 1, p. 2-13, jan./fev. 2001.

MINGHELLI, Beatriz et al. Comparação dos níveis de ansiedade e depressão entre idosos ativos e sedentários. Revista de Psiquiatria Clínica, São Paulo, v. 40, n. 2 , p. $71-76,2013$.

MOTA, Jorge et al. Atividade física e qualidade de vida associada à saúde em idosos participantes e não participantes em programas regulares de atividade física. Revista Brasileira de Educação Física e Esporte, São Paulo, v. 20, n. 3, p. 219-225, jul./set. 2006.

PENEDO, Frank J.; DAHN, Jason R. Exercise and Well-Being: A Review of Mental and Physical Health Benefits Associated with Physical Activity. Current Opinion in Psychiatry, Philadelphia, PA, v. 18, n. 2, p. 189-193, Mar. 2005

PHROMPAET, Sureeporn et al. Effects of Pilates Training on Lumbo-Pelvic Stability and Flexibility. Asian Journal of Sports Medicine, Tehran, v. 2, n. 1, p. 16-22, Mar. 2011.

POLLOCK, Michael L. et al. Resistance Exercise in Individuals with and without Cardiovascular Disease. Circulation, Dallas, TX, v. 101, n. 7, p. 828-833, Feb. 2000

ROGERS, Kate; GIBSON, Ann L. Eight-Week Traditional Mat Pilates Training-Program Effects on Adult Fitness Characteristics. Research Quarterly for Exercise and Sport, Washington, D.C., v. 80, n. 3, p. 569-574, Sept. 2009.

SCHULZ, Kenneth F.; ALTMAN, Douglas G.; MOHER, David. CONSORT 2010 Statement: updated guidelines for reporting parallel group randomised trials. Obstetrics and Gynecology, New York, v. 115, n. 5, p. 1063-1070, May 2010.

SIQUEIRA RODRIGUES, Brena Guedes de et al. Pilates Method in Personal Autonomy, Static Balance and Quality of Life of Elderly Females. Journal of Bodywork and Movement Therapies, New York, v. 14, n. 2, p. 195-202, Apr. 2010.

TAVARES, Darlene Mara dos Santos; DIAS, Flavia Aparecida. Capacidade funcional, morbidades e qualidade de vida de idosos. Texto \& Contexto: Enfermagem, Florianópolis, v. 21, n. 1, p. 112-120, jan./mar. 2012.

THE WHOQOL GROUP. Development of the World Health Organization WHOQOL-bref quality of life assessment. Psychological Medicine, London, v. 28, n. 3, p. 551-558, May 1998. 